\title{
NUEVOS SITIOS CON REPRESENTACIONES RUPESTRES EN LA LOCALIDAD POTROK AIKE (SANTA CRUZ, ARGENTINA)
}

JUDITH CHARLIN"

\section{RESUMEN}

Se presentan nuevos datos sobre la distribución de representaciones rupestres en la localidad Potrok Aike (Santa Cruz, Argentina), donde se relevaron distintas concentraciones de motivos y motivos aislados en los afloramientos volcánicos localizados hacia el noroeste de la laguna. Se trata de pinturas predominantemente en rojo, con escasos motivos en blanco. Las representaciones abstractas son mayoritarias, aunque los positivos de manos ocupan el segundo lugar de abundancia entre los tipos de motivos.

Las diferencias tonales registradas (dos series tonales en rojo), la superposición de motivos y el repintado sugieren distintos momentos de ejecución, que de acuerdo con los antecedentes del área corresponderían al Holoceno tardío. Así, se describe la forma, tamaño, color y tonalidad, tratamiento técnico y distribución de los motivos y se realiza una comparación regional mostrando las principales tendencias de las representaciones rupestres en el campo volcánico Pali Aike.

PALABRAS CLAVE: pinturas rupestres, distribución, Potrok Aike

\section{ABSTRACT}

New data about rock art distribution in Potrok Aike locality (Santa Cruz Province, Argentina) is presented. Motif concentrations and isolated motifs were surveyed on volcanic outcrops located to the northwest of the Potrok Aike lagoon. They are paintings, mainly in red, with a few motifs in white. Most of them are abstract representations, although hand stencils are the second in abundance.

Different tonalities (two red tones), superposition and repainting suggest distinct times of painting execution, which according to the regional background correspond to Late Holocene. Shape, size, color and tonality, technical treatment and motifs distribution are described. Finally, a regional comparison shows the main trends in Pali Aike volcanic field rock art.

KEYWORDS: rock art paintings, distribution, Potrok Aike 
INTRODUCCIÓN:

ANTECEDENTES REGIONALES

Afortunadamente, en el tiempo reciente se han dado a conocer nuevos datos y análisis comparativos de las representaciones rupestres en el extremo sur de Patagonia meridional (Campan et al. 2007; Charlin \& Borrero 2012; Fiore 2006; Manzi \& Carballo Marina 2012; Sepúlveda 2011). Sin embargo, desde las primeras descripciones efectuadas por O. Menghin $(1952,1957)$ y el Padre M. Molina (1969, 1969-1970, 1972, 1976), el arte rupestre del extremo austral de Patagonia, en particular del campo volcánico Pali Aike (CVPA), solo ha recibido escasa atención, al menos en territorio Argentino (Hernández Llosas et al. 1999; Gómez Otero 1986-1987, 1989-1990; Sanguinetti de Bórmida 1976, 1982). En la porción Chilena del CVPA y en la región de Ultima Esperanza (Magallanes, Chile), los estudios pioneros de Bate (1970, 1971) llevaron a la definición de un estilo particular para el sur de Patagonia, denominado "estilo Río Chico" en referencia a la abundancia de sitios con representaciones rupestres en la cuenca de este río. Dicho estilo luego fue segmentado en "modalidades estilísticas o subestilos" de acuerdo con los estudios posteriores de Massone (1982:92). Los relevamientos de Prieto (1989-1990, 1997) en el paralelo $52^{\circ}$, de Prieto y coautores (1998) en Cañadón Leona y recientemente los de Gallardo (2009) en la cuenca del río Chico completan el registro de sitios con arte rupestre en el extremo austral de Patagonia (para una descripción más completa de los antecedentes ver Charlin \& Borrero 2012).

Los estudios pioneros han caracterizado, de manera cualitativa, el arte rupestre del sur de Patagonia meridional por el predominio de motivos abstractos geométricos simples y se ha sostenido su uniformidad en espacios amplios, abarcando sectores de Argentina y Chile (Bate 1970; Menghin 1957). Si bien Bate (1970:22) postula un área de dispersión menor a la sostenida por Menghin (1957:77) bajo su "estilo de símbolos complicados" que cubre prácticamente toda Patagonia, señala que Los motivos del arte rupestre en la Patagonia chilena son exactamente los mismos que aparecen en la argentina, correspondiendo, por lo tanto, al área de dispersión de los mismos pueblos de cazadores nómadas, con sólo algunas posibles diferencias culturales (...), señalando conexiones entre la Prov. de Magallanes en la Patagonia chilena, Punta Gualichu en lago Argentino (Prov. Santa Cruz, Argentina) y el Río Pinturas (Prov. Santa Cruz, Argentina). Los estudios recientes que han intentado identificar patrones en la distribución del arte, tratando de cuantificar de algún modo la información previa, han mostrado que las similitudes, en una escala amplia, son más relativas que absolutas (Charlin \& Borrero 2012; Manzi \& Carballo Marina 2012), y que "las posibles diferencias culturales" señaladas por Bate (1970) aparecen avaladas también por otras líneas de evidencia como la distribución de las materias primas líticas utilizadas para la manufactura de artefactos y las evidencias arqueofaunísticas y de isótopos estables sobre restos óseos humanos que permiten caracterizar la dieta de los grupos humanos (Borrazzo \& Charlin 2012; Charlin \& Borrero 2012).

A través de un Análisis de Componentes Principales (PCA) sobre una matriz de presenciaausencia de tipos de motivos por área, Charlin \& Borrero (2012) han mostrado tendencias diferenciales en la abundancia de tipos de motivos y en los procedimientos técnicos entre el CVPA, Ultima Esperanza y lago Argentino. Mientras en el primero predominan los trazos y motivos geométricos lineales, en Ultima Esperanza lo hacen los puntos y motivos geométricos puntiformes. A diferencia de estos espacios donde las representaciones figurativas se encuentran ausentes o son escasas, hacia lago Argentino los motivos figurativos (antropomorfos y zoomorfos) aumentan proporcionalmente. Además, en cuanto a los procedimientos técnicos, mientras en el CVPA y Ultima Esperanza solo se registran pinturas, en lago Argentino también hay representaciones grabadas (contra Fiore 2006). Por consiguiente, más allá de un sustrato común caracterizado por la pintura de motivos abstractos geométricos, la técnica de realización y las proporciones de tipos de motivos son diferentes en las tres regiones. Asimismo, Manzi \& Carballo Marina (2012), también trabajando sobre una matriz de presenciaausencia de tipos de motivos, encuentran diferencias en la diversidad de los conjuntos entre sitios y entre áreas, al comparar la cuenca del río 
Gallegos contra el río Chico y Ultima Esperanza.

En este marco, se presenta la información relevada sobre las representaciones rupestres en dos sectores de una meseta volcánica localizada hacia el NO de la laguna Potrok Aike (CVPA, Prov. Santa Cruz, Argentina), pretendiendo contribuír a extender la discusión sobre los patrones de representación de las pinturas rupestres en el CVPA.

\section{LAS REPRESENTACIONES RUPESTRES EN POTROK AIKE: ANTECEDENTES LOCALES}

Los trabajos publicados por Gómez Otero (1986-1987, 1987, 1993) son los que han arrojado la primera información arqueológica sobre las ocupaciones humanas en la laguna Potrok Aike. Así, en el alero 1 se identificaron tres series de niveles culturales, entendidos como un único componente. Una lente carbonosa entre las ocupaciones medias y tardías arrojó una fecha de $740 \pm 180{ }^{14} \mathrm{C}$ años AP (Gómez Otero 19861987, 1987). Un trozo de hierro recuperado en los niveles tardíos señala al menos una ocupación en momentos históricos (Gómez Otero 1986-1987).

Recientemente, se han efectuado nuevos relevamientos en la laguna persiguiendo objetivos tafonómicos y estudiando la disponibilidad de materias primas líticas (Borrero et al. 2013; Charlin 2009; Charlin \& Pallo 2013). Se realizaron sondeos en médanos de deflación en la margen este de la laguna, uno de los cuales arrojó una edad de $4.879 \pm 58$ AP (Borrero et al. 2013), lo cual extiende las primeras ocupaciones de la laguna al Holoceno medio.

Lamentablemente, los relevamientos de las representaciones rupestres de la cueva 1 y de un reparo en el extremo oriental del farallón volcánico que rodea a la laguna en su margen sur, no fueron publicados (Gómez Otero 1983-85).

En la cueva 1, Gómez Otero (1983-85) señala la presencia de pinturas sobre las paredes externas, entre 1,35 y $1,5 \mathrm{~m}$ de altura del suelo actual. Se trata de cuatro motivos en dos tonalidades de rojo: dos motivos abstractos (dos circunferencias yuxtapuestas en rojo claro y un triángulo isósceles

1 Dicho reparo es denominado por Manzi \& Carballo Marina (2012:tabla 2) como alero 1 (PAA1). No se debe confundir con el alero 1 excavado por Gómez Otero (1986-1987) y en rojo subido) y dos figurativos (un zoomorfo semejante a un matuasto en rojo claro y un motivo compuesto por tres tridígitos en negro sobre fondo preparado en rojo subido). La superposición del triángulo en rojo subido sobre el posible matuasto en rojo claro nos señala la existencia de diferentes momentos de ejecución y por consiguiente de diferencias diacrónicas. Gómez Otero (198385:58) señala que el color rojo del triángulo y del fondo preparado para los tridígitos negros son del mismo tono, por lo cual considera al rojo subido y al negro como contemporáneos y disociados del rojo claro.

En el reparo en margen sur (en adelante reparo $\left.{ }^{1}\right)$, la cantidad y variedad de motivos es mayor, aunque el conjunto sigue siendo pequeño. Se trata de ocho motivos abstractos (circunferencias, trazos, cuadrángulo, greca o laberintiforme) y cuatro figurativos (tres antropomorfos y un posible guanaco), todos en rojo claro.

En este conjunto en particular llama la atención la presencia de antropomorfos (1), de un posible guanaco (2) y de una greca o laberintiforme (3), como se indica a continuación.

(1) En los sitios con arte rupestre de la cuenca del río Gallegos, los antropomorfos no son un motivo frecuente; solo recientemente se ha reportado la presencia de tres antropomorfos en el Puesto Cañadón Verde (Estancia Bella Vista, Manzi \& Carballo Marina 2012), sobre un total de 15 sitios con pinturas rupestres registrados en esta cuenca (incluyendo los aquí presentados). Los antropomorfos son más abundantes en el río Chico, donde se menciona su existencia en Markatch Aike (Molina 1972), Cañadón Seco 1, Cañadón Leona, Río Chico 1 y 2 y Ush Aike (Bate 1970, 1971).

En Potrok Aike los antropomorfos son caracterizados como estilizado uno de ellos y los otros dos como complicados y semejantes al que Luna Pont (1976 citado en Gómez Otero 19831985) describió en Lago Roca III (Lago Argentino). Se señala además que estos dos motivos poseen tocado (Gómez Otero 1983-85). Nuestro estudio previo ha mostrado que los motivos figurativos en general y los antropomorfos en particular muestran un incremento hacia el NO, en Ultima Esperanza

mencionado al principio del apartado. Nosotros conservamos el término de "reparo" debido a que Gómez Otero (19831985) originariamente lo denomina de esta manera. 
y especialmente en la zona de lago Argentino (Charlin \& Borrero 2012). Estas diferencias en la distribución de determinado tipo de motivo están sugiriendo diferencias regionales (que posiblemente pueden ser temporales).

(2) Con respecto a los zoomorfos en general, más allá de los "rastros de avestruz" , en el CVPA y Ultima Esperanza solo se cuenta con referencias a zoomorfos indeterminados (en cinco casos), a saber en Romario Barría (Campan et al. 2007), Río Chico 2 (Bate 1971), Cerro Benitez 2 (Massone 1982), Lago Sofía 2 (Massone 1982) y Puesto El Cóndor, donde un tridígito fue reciclado como zoomorfo (sin especificación, Manzi \& Carballa Marina 2012), siendo identificado un felino en lago Sarmiento 2 (Bate 1971:34). Representaciones de guanacos identificados como tales están completamente ausentes, como así también los matuastos. La única referencia existente es la señalada anteriormente en la cueva 1 de Potrok Aike (Gómez Otero 1983-85).

(3) Las grecas o laberintiformes no han sido registrados en lo que Bate $(1970,1971)$ originalmente definió como el "estilo Río Chico". Sin embargo, posteriormente se ha mencionado la presencia de motivos descriptos de este modo en sitios del CVPA asignados a este estilo (un "laberinto" en Romario Barría, Campan et al. 2007, y representaciones del "Estilo de grecas" en Markatch Aike, Molina 1972: fig. 18).

Consideramos que la presencia de este tipo de representaciones, junto con los antropomorfos y guanacos señalados anteriormente, podrían estar señalando que el conjunto del reparo es muy tardío, aludiendo a momentos históricos (Belardi 2004). De hecho, los laberintos formados comúnmente por una sola linea ininterrumpida (...), pero a veces también por el espacio o corredor entre dos lineas (...) son considerados un tipo de motivo tardío dentro del Estilo de Grecas definido por Menghin (1957:72). Con respecto a los momentos tardíos, Gómez Otero (198385) señala que según Casamiquela las crónicas etnohistóricas mencionan el uso de tocado entre los Tehuelches, en relación con la presencia del mismo en los motivos antropomorfos.

\footnotetext{
2 Que sería un caso de metonimia, es decir, la representación
}

\section{NUEVOS SITIOS CON REPRESENTACIONES RUPESTRES EN POTROK AIKE}

En la meseta volcánica localizada hacia el noroeste de la laguna se relevaron distintas concentraciones de representaciones rupestres distribuidas a lo largo del paredón oeste y en una serie de aleros ubicados en el flanco norte (Fig. 1).

En el paredón occidental, denominado localmente como "Potrero de las pinturas" (PP), se relevaron siete concentraciones ( $\mathrm{C} 1$ a 7) y dos motivos aislados (MA1 y 2) a lo largo de $200 \mathrm{~m}$ en colores rojo y blanco, lo cual suma un total de 69 motivos. La mala conservación de las pinturas debido a la insolación y exfoliación de la roca sugiere que el número podría ser mayor.

Hacia el norte del afloramiento, por el camino de entrada al INTA, se registraron pinturas rupestres en dos reparos de la colada basáltica. En este caso el afloramiento volcánico no presenta las características de un paredón, sino que es rugoso y se encuentra conformado por la sucesión de varios reparos de pequeñas dimensiones, muchos de los cuales se encuentran derruidos. Las paredes de basalto presentan exfoliaciones y fisuras, como así también la presencia frecuente de líquenes, lo que ha afectado fuertemente la conservación de las pinturas. En el sitio 1 (S1) se han relevado un total de 23 motivos distribuidos en tres concentraciones sobre paneles con distinta orientación. En el sitio 2 (S2), solo se registra la asociación de dos motivos en la $\mathrm{C} 1$ y un motivo aislado en un panel independiente. La totalidad de las representaciones de los sitios 1 y 2 son en rojo.

De cada concentración o motivo aislado se registraron las coordenadas geográficas, la altura de los motivos respecto al nivel actual del suelo y la distancia lateral entre los mismos. En los casos en los que las representaciones se encontraban a gran altura (como en la C2, ver infra), lo cual impedía el registro de su posición, se trabajó en laboratorio con el programa tpsDIG2 (Thin Plate Spline Digitizing versión 2.16, Rohlf 2010) para obtener las distancias relativas entre motivos. Dicho programa permite escalar la imagen, y así posteriormente tomar medidas métricas y angulares entre los motivos y de los motivos mismos, lo cual posibilita alcanzar un alto grado de detalle.

de la parte por el todo. 




Fig.1. Localización del Potrero de las pinturas (PP) y de los sitios 1 (S1) y 2 (S2) en la localidad Potrok Aike
Para la descripción formal y técnica de los motivos rupestres y su posterior cuantificación se siguió la guía para el relevamiento y clasificación de las representaciones rupestres propuesta por Hernández Llosas (1985). Con respecto a la denominación de los motivos, en los casos en los que fue posible se utilizaron las categorías propuestas por Massone (1982) y Manzi \& Carballo Marina (2012).

\section{Potrero de las pinturas}

Las concentraciones presentan un mínimo de dos motivos y un máximo de 28 , teniendo la mayoría menos de 10 motivos (tabla 1). Los tipos de motivos más abundantes son las series de trazos paralelos $(n=34)$, todos en color rojo, y los positivos de mano, en su mayoría en rojo $(n=8)$ aunque también se registran algunos ejemplares en blanco $(\mathrm{n}=3)$. Fuera de los dos tipos de motivos predominantes, las demás categorías $(n=15)$ solo se encuentran representadas por uno o dos motivos, excepto los trazos aislados que suman cuatro (tabla 2).

Se destacan en particular las C2 y C8 por ser las que presentan las mayores frecuencias de series de trazos paralelos $(\mathrm{n}=26)$ y positivos de manos $(n=5)$ respectivamente. Asimismo, presentan evidencias que sugieren diferentes momentos de ejecución de las pinturas.

La C2 reviste particular importancia en tanto se encuentra ubicada en un gran panel lateral a la boca de entrada a una cueva $\left(70^{\circ} 26^{\prime} 36^{\prime \prime} \mathrm{W}\right.$ $51^{\circ} 56^{\prime} 41.5^{\prime \prime}$ ) que presenta abundante material en superficie (ver fig. 22.2 en Charlin \& Borrero 2012:386). En esta cueva se registra, además, otra concentración sobre la boca de entrada (C5) y también una en el vestíbulo (C6).

En la C2, compuesta por 26 motivos de trazos paralelos, un trazo aislado y un óvalo con diseño interno o "vulva", es posible diferenciar dos series tonales (Aschero 1979) en rojo que no dependen de la exposición. Asimismo se observan casos de repintado en el sector del panel que se encuentra más expuesto a la insolación. A igualdad de exposición los motivos ejecutados con un mismo tono pero con diferentes grados de conservación o intensidad tonal son considerados diacrónicos, es decir representan tiempos de exposición diferentes (Aschero 1979: 425).

La parte inferior del panel se encuentra fragmentada, encontrándose el fragmento de roca 
Tabla 1. Frecuencias de motivos según categorías formales y técnicas por sitio, concentración y motivo aislado.

Referencias: $\mathrm{mot}=$ motivo, $\mathrm{sim}=$ simple, $\mathrm{com}=\mathrm{compuesto}$, abs=abstracto, fig=figurativo, indif=indiferenciado, lin=lineal, estam=estampado, estar=estarcido, comb=combinado, rec=rectilíneo, cur=curvilíneo, desv=desvaído, osc=oscuro, bl=blanco.

\begin{tabular}{|c|c|c|c|c|c|c|c|c|c|c|c|c|c|c|c|c|c|c|c|}
\hline & \multirow[b]{2}{*}{$\begin{array}{c}\mathrm{n}^{\circ} \\
\text { mot }\end{array}$} & \multicolumn{2}{|c|}{ Tipo mot } & \multicolumn{3}{|c|}{ Forma } & \multicolumn{5}{|c|}{ Tratamiento técnico } & \multicolumn{3}{|c|}{ Trazo lineal } & \multicolumn{4}{|c|}{ Color y tono } \\
\hline & & & $\operatorname{sim}$ & com & abs & fig & indif & $\operatorname{lin}$ & estam & estar & comb & indif & rec & cur & comb & $\begin{array}{l}\text { rojo } \\
\text { desv }\end{array}$ & $\begin{array}{l}\text { rojo } \\
\text { osc }\end{array}$ & rep* & $\mathrm{bl}$ \\
\hline \multirow{11}{*}{$\begin{array}{l}0 \\
0 \\
\Xi \\
. \\
.0 \\
0 \\
0 \\
0 \\
0 \\
0 \\
0 \\
0 \\
0 \\
0 \\
0\end{array}$} & $\mathrm{C} 1$ & 9 & 7 & 2 & 9 & 0 & 0 & 9 & 0 & 0 & 0 & 0 & 6 & 1 & 2 & 9 & 0 & 0 & 0 \\
\hline & MA1 & 1 & 1 & 0 & 0 & 1 & 0 & 1 & 0 & 0 & 0 & 0 & 1 & 0 & 0 & 1 & 0 & 0 & 0 \\
\hline & $\mathrm{C} 2$ & 28 & 2 & 26 & 28 & 0 & 0 & 28 & 0 & 0 & 0 & 0 & 27 & 1 & 0 & 10 & 15 & 3 & 0 \\
\hline & $\mathrm{C} 3$ & 2 & 2 & 0 & 2 & 0 & 0 & 2 & 0 & 0 & 0 & 0 & 1 & 1 & 0 & 2 & 0 & 0 & 0 \\
\hline & $\mathrm{C} 4$ & 10 & 5 & 5 & 9 & 1 & 0 & 9 & 1 & 0 & 0 & 0 & 7 & 2 & 0 & 9 & 1 & 0 & 0 \\
\hline & C5 & 3 & 3 & 0 & 0 & 3 & 0 & 0 & 3 & 0 & 0 & 0 & 0 & 0 & 0 & 3 & 0 & 0 & 0 \\
\hline & C6 & 4 & 4 & 0 & 2 & 0 & 2 & 2 & 0 & 0 & 0 & 2 & 1 & 1 & 0 & 4 & 0 & 0 & 0 \\
\hline & MA2 & 1 & 1 & 0 & 1 & 0 & 0 & 1 & 0 & 0 & 0 & 0 & 1 & 0 & 0 & 1 & 0 & 0 & 0 \\
\hline & C7 & 4 & 3 & 1 & 2 & 2 & 0 & 2 & 2 & 0 & 0 & 0 & 2 & 0 & 0 & 4 & 0 & 0 & 0 \\
\hline & $\mathrm{C} 8$ & 7 & 7 & 0 & 2 & 5 & 0 & 2 & 5 & 0 & 0 & 0 & 2 & 0 & 0 & 4 & 0 & 0 & 3 \\
\hline & Total & 69 & 35 & 34 & 55 & 12 & 2 & 0 & 11 & 0 & 0 & 2 & 48 & 6 & 2 & 47 & 16 & 3 & 3 \\
\hline \multirow{4}{*}{ 골 } & C1 & 17 & 15 & 2 & 14 & 3 & 0 & 14 & 2 & 0 & 1 & 0 & 10 & 3 & 2 & 17 & 0 & 0 & 0 \\
\hline & $\mathrm{C} 2$ & 4 & 4 & 0 & 4 & 0 & 0 & 0 & 0 & 3 & 1 & 0 & 0 & 0 & 1 & 4 & 0 & 0 & 0 \\
\hline & C3 & 2 & 2 & 0 & 0 & 2 & 0 & 0 & 2 & 0 & 0 & 0 & 0 & 0 & & 1 & 1 & 0 & 0 \\
\hline & Total & 23 & 21 & 2 & 18 & 5 & 0 & 14 & 4 & 3 & 2 & 0 & 10 & 3 & 3 & 22 & 1 & 0 & 0 \\
\hline \multirow{3}{*}{$\begin{array}{l}N \\
\stackrel{\circ}{*} \\
\text { ஸे }\end{array}$} & $\mathrm{C} 1$ & 2 & 0 & 2 & 2 & 0 & 0 & 2 & 0 & 0 & 0 & 0 & 2 & 0 & 0 & 0 & 2 & 0 & 0 \\
\hline & MA & 1 & 0 & 1 & 1 & 0 & 0 & 1 & 0 & 0 & 0 & 0 & 1 & 0 & 0 & 0 & 1 & 0 & 0 \\
\hline & Total & 3 & 0 & 3 & 3 & 0 & 0 & 3 & 0 & 0 & 0 & 0 & 3 & 0 & 0 & 0 & 3 & 0 & 0 \\
\hline
\end{tabular}

*Repintado rojo oscuro sobre rojo desvaído

desprendido al pie del paredón. Esto ha provocado que la hilera inferior de motivos $(n=5)$ se encuentre cortada. Su contraparte en el bloque desprendido se encuentra exfoliada, posiblemente por huaqueo. Tanto esta cicatriz, como el resto de la cara desprendida se encuentran cubiertas por grandes líquenes.

Los motivos de trazos paralelos, también denominados "arrastre de dedos", se encuentran compuestos por diferente cantidad de elementos (Gradin 1978), teniendo como mínimo dos trazos y como máximo 13, siendo los más frecuentes los de 3 $(n=5), 5(n=6)$ y $7(n=5)$ trazos. El largo promedio de los trazos es de $2,83 \mathrm{~cm}$, con un mínimo de $2,4 \mathrm{~cm}$ y un máximo de $3,86 \mathrm{~cm}$, siendo el ancho promedio de $1,6 \mathrm{~cm}$, con $1,3 \mathrm{~cm}$ y $2 \mathrm{~cm}$ como valores mínimo y máximo respectivamente.

Cabe señalar que en los casos de trazos aislados (motivos simples), la longitud siempre es mayor (de 9 a $28 \mathrm{~cm}$ ). Asimismo esto se cumple en los casos de series de trazos paralelos que han sido realizadas utilizando como eje de la representación alguna arista del afloramiento volcánico ( $n=2$ ) (Fig. 2). Estos son los únicos casos en los que el alineamiento de los trazos es en sentido vertical.

La C8 presenta asociados espacialmente cinco

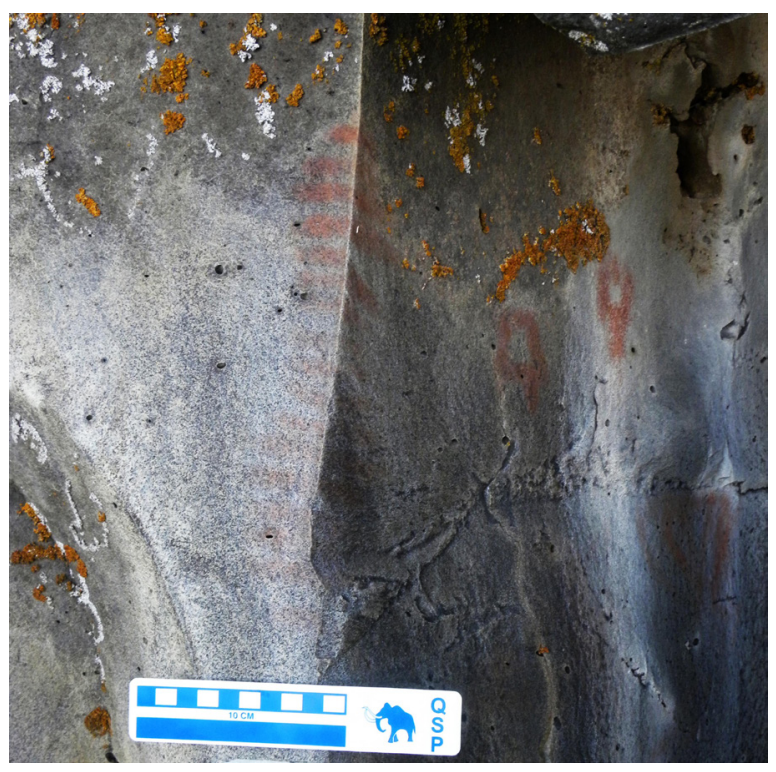

Fig.2. Serie de trazos paralelos usando como eje una arista del soporte (Potrero de las pinturas $\mathrm{C} 1$ )

positivos de manos y dos motivos abstractos (un trazo y un cruciforme). Los motivos están realizados en dos colores, rojo y blanco. En rojo encontramos dos manos y los dos motivos abstractos. Los tres 
Tabla 2. Descripción y frecuencia de los motivos por sitio, concentración y motivo aislado

\begin{tabular}{|c|c|c|c|c|c|c|c|c|c|c|c|c|c|c|c|c|c|c|}
\hline \multirow[b]{2}{*}{ Descripción motivos } & \multicolumn{11}{|c|}{ Potrero de las pinturas (paredón oeste) } & \multicolumn{4}{|c|}{ Sitio 1 (flanco norte) } & \multicolumn{3}{|c|}{ Sitio 2 (flanco norte) } \\
\hline & $\mathrm{C} 1$ & MA1 & $\mathrm{C} 2$ & C3 & $\mathrm{C} 4$ & C5 & C6 & MA2 & $\mathrm{C} 7$ & $\mathrm{C} 8$ & total & $\mathrm{C} 1$ & $\mathrm{C} 2$ & $\mathrm{C} 3$ & total & $\mathrm{C} 1$ & MA & Total \\
\hline Trazo aislado & 1 & 0 & 1 & 1 & 0 & 0 & 1 & 1 & 0 & 1 & 6 & 3 & 0 & 0 & 3 & 0 & 0 & 0 \\
\hline Serie de trazos paralelos & 2 & 0 & 26 & 0 & 5 & 0 & 0 & 0 & 1 & 0 & 34 & 0 & 0 & 0 & 0 & 2 & 1 & 3 \\
\hline Trazos radiados & 0 & 0 & 0 & 0 & 0 & 0 & 0 & 0 & 0 & 0 & 0 & 1 & 0 & 0 & 1 & 0 & 0 & 0 \\
\hline Trazo horiz c/apéndice ${ }^{\mathrm{a}}$ & 0 & 0 & 0 & 0 & 0 & 0 & 0 & 0 & 0 & 0 & 0 & 1 & 0 & 0 & 1 & 0 & 0 & 0 \\
\hline Trazos y cruces & 0 & 0 & 0 & 0 & 0 & 0 & 0 & 0 & 0 & 0 & 0 & 1 & 0 & 0 & 1 & 0 & 0 & 0 \\
\hline Semicírculo de cruces & 0 & 0 & 0 & 0 & 0 & 0 & 0 & 0 & 0 & 0 & 0 & 1 & 0 & 0 & 1 & 0 & 0 & 0 \\
\hline Cruciforme & 0 & 0 & 0 & 0 & 0 & 0 & 0 & 0 & 0 & 1 & 1 & 2 & 0 & 0 & 2 & 0 & 0 & 0 \\
\hline Triángulo & 1 & 0 & 0 & 0 & 1 & 0 & 0 & 0 & 0 & 0 & 2 & 0 & 0 & 0 & 0 & 0 & 0 & 0 \\
\hline Rectángulo & 2 & 0 & 0 & 0 & 0 & 0 & 0 & 0 & 0 & 0 & 2 & 0 & 0 & 0 & 0 & 0 & 0 & 0 \\
\hline Rectángulo c/diseño interno ${ }^{b}$ & 0 & 0 & 0 & 0 & 0 & 0 & 0 & 0 & 1 & 0 & 1 & 0 & 0 & 0 & 0 & 0 & 0 & 0 \\
\hline Rectángulo arqueado c/apéndice & 0 & 0 & 0 & 0 & 0 & 0 & 0 & 0 & 0 & 0 & 0 & 1 & 0 & 0 & 1 & 0 & 0 & 0 \\
\hline Cuadrángulo & 0 & 0 & 0 & 0 & 1 & 0 & 0 & 0 & 0 & 0 & 1 & 0 & 0 & 0 & 0 & 0 & 0 & 0 \\
\hline Circunferencia (Circunf) & 0 & 0 & 0 & 0 & 1 & 0 & 1 & 0 & 0 & 0 & 2 & 1 & 0 & 0 & 1 & 0 & 0 & 0 \\
\hline Circunf c/ apéndice & 2 & 0 & 0 & 0 & 0 & 0 & 0 & 0 & 0 & 0 & 2 & 0 & 0 & 0 & 0 & 0 & 0 & 0 \\
\hline Circunf $\mathrm{c} /$ punto central & 0 & 0 & 0 & 0 & 0 & 0 & 0 & 0 & 0 & 0 & 0 & 1 & 0 & 0 & 1 & 0 & 0 & 0 \\
\hline Circunf radiada c/punto central & 0 & 0 & 0 & 0 & 0 & 0 & 0 & 0 & 0 & 0 & 0 & 1 & 0 & 0 & 1 & 0 & 0 & 0 \\
\hline Círculo radiado & 0 & 0 & 0 & 0 & 0 & 0 & 0 & 0 & 0 & 0 & 0 & 1 & 1 & 0 & 2 & 0 & 0 & 0 \\
\hline Óvalo & 0 & 0 & 1 & 0 & 0 & 0 & 0 & 0 & 0 & 0 & 1 & 0 & 0 & 0 & 0 & 0 & 0 & 0 \\
\hline Óvalo c/diseño interno ${ }^{c}$ & 0 & 0 & 0 & 0 & 1 & 0 & 0 & 0 & 0 & 0 & 1 & 0 & 0 & 0 & 0 & 0 & 0 & 0 \\
\hline Almena & 1 & 0 & 0 & 0 & 0 & 0 & 0 & 0 & 0 & 0 & 1 & 0 & 0 & 0 & 0 & 0 & 0 & 0 \\
\hline U invertida & 0 & 0 & 0 & 1 & 0 & 0 & 0 & 0 & 0 & 0 & 1 & 0 & 0 & 0 & 0 & 0 & 0 & 0 \\
\hline Tridígito & 0 & 1 & 0 & 0 & 0 & 0 & 0 & 0 & 0 & 0 & 1 & 1 & 0 & 0 & 1 & 0 & 0 & 0 \\
\hline Positivo de mano & 0 & 0 & 0 & 0 & 1 & 3 & 0 & 0 & 2 & 5 & 11 & 2 & 0 & 2 & 4 & 0 & 0 & 0 \\
\hline Mancha & 0 & 0 & 0 & 0 & 0 & 0 & 0 & 0 & 0 & 0 & 0 & 0 & 3 & 0 & 3 & 0 & 0 & 0 \\
\hline Indif. por conservación & 0 & 0 & 0 & 0 & 0 & 0 & 2 & 0 & 0 & 0 & 2 & 0 & 0 & 0 & 0 & 0 & 0 & 0 \\
\hline total & 9 & 1 & 28 & 2 & 10 & 3 & 4 & 1 & 4 & 7 & 69 & 17 & 4 & 2 & 23 & 2 & 1 & 3 \\
\hline
\end{tabular}

a el apéndice es un trazo vertical corto en el medio del trazo horizontal

b también denominado genéricamente "rectángulo con diseño geométrico" o "cartucho" por Aschero et al. 2006 o específicamente "cartucho reticulado" en referencia a una de las variantes particulares de esa clase

c comúnmente denominado "vulva", sobre todo en la arqueología del Viejo Mundo

motivos restantes son manos en blanco, una de ellas superpuesta al motivo cruciforme en rojo.

De acuerdo a la totalidad de motivos relevados en el sitio, la cantidad de motivos simples y compuestos es similar, siendo estos últimos mayoría solo por una unidad (34 y 35 respectivamente). Esta no parece ser la tendencia regional, de acuerdo con los escasos trabajos que presentan información cuantitativa al respecto (Campan et al. 2007; Manzi \& Carballo Marina 2012). De hecho, el elevado número de motivos compuestos obedece a la abundancia de

3 Cabe señalar que los motivos de forma oval con una línea en el medio comúnmente denominados "vulvas", series de trazos paralelos en la C2.

La proporción de motivos simples y compuestos no sigue una distribución uniforme por concentración (Fig. 3), siendo los primeros los que en general predominan. Los motivos compuestos están ausentes en cuatro concentraciones y en las restantes solo son mayoría en la C2. Asimismo, los dos motivos aislados son simples.

Los motivos abstractos son los predominantes, representando el $80 \%$ del total $(n=55)^{3}$. Nuevamente se destaca en abundancia

son considerados como motivos abstractos a pesar de su denominación figurativa. 




Fig.3. Frecuencia de motivos simples y compuestos en Potrero de las pinturas

la C2. Se trata de formas geométricas simples, similares a aquellas que Bate (1970, 1971) consideró para definir el "estilo Río Chico", tales como trazos, triángulos, círcunferencias con apéndices, almenas, rectángulos, entre otros (ver tabla 2).

Los motivos figurativos representan el $17 \%(n=12)$ y son tan solo manos y un tridígito. Mientras entre los motivos abstractos encontramos diversidad de representaciones (15 tipos de motivos identificados), los motivos figurativos se reducen a dos tipos. Las diferencias en la diversidad entre ambas clases de motivos son notables, como lo han señalado Manzi \& Carballo Marina (2012).

Con respecto a las manos, se registran tres casos de manos izquierdas y tres de manos derechas, siendo las restantes indiferenciadas por mala conservación. Asimismo, es posible diferenciar dos conjuntos según tamaño máximo. En las C4 y 5, las manos muestran un largo de 18,20 y $30 \mathrm{~cm}$ siendo todas ellas de color rojo desvaído. En las C7 y 8, los tamaños son menores (Fig. 4), habiendo cuatro casos con un largo de $14 \mathrm{~cm}$ (en rojo desvaído y en blanco) y dos de $15 \mathrm{~cm}$ (en rojo desvaído). Positivos de manos también han sido registrados en Güer Aike (Molina 1972), Abrigo de los Pescadores (Molina 196970, 1972), Rose Aike 3 (Massone 1982) y Puesto El Cóndor (Manzi \& Carballo Marina 2012). Lamentablemente sólo conocemos la frecuencia para este último sitio, siendo un solo motivo (Manzi \& Carballo Marina 2012). Los negativos de manos son menos frecuentes regionalmente, pero han sido identificados en un alero cercano a la Cueva de los Contrabandistas (Prieto 1989-90), Río Chico 5 (Bate 1970), Güer Aike y Puesto Cañadón Verde (Manzi \& Carballo Marina 2012).

En cuanto al tratamiento técnico, predominan los motivos lineales ( $n=56,81 \%)$, de los cuales el $87 \%$ son rectilíneos $(n=48)$. El resto de los motivos son estampados (excepto dos indiferenciados), correspondiendo todos ellos a positivos de manos. Cabe señalar que por tratamiento técnico nos referimos a los aspectos tecnológicos correspondientes a los procesos de manufactura; en el caso particular de las pinturas

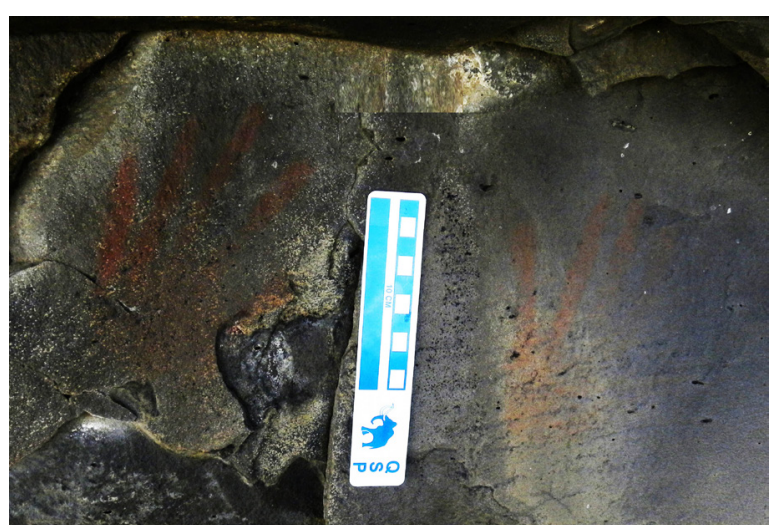

Fig.4. Positivos de manos en Potrero de las pinturas (C7) 
aquí descriptas, siguiendo a Hernández Llosas (1985:16), diferenciamos si la representación ha sido realizada mediante trazos continuos -lineal-, discontinuos -puntiforme-, mediante el llenado de una superficie -plano- o una combinación de dos o más técnicas. A estas tres categorías hemos sumado la de "estampado" para los casos de positivos y la de "estarcido" para el caso de manchas y negativos (Bate 1970, 1971), dos procedimientos técnicos usuales en la Patagonia Austral. Entendemos que el uso de la categoría "tratamiento plano" por Campan et al. (2007) y Manzi \& Carballo Marina (2012) responde a otro concepto o clasificación, dado que en general se están refiriendo a lo que nosotros denominamos como tratamiento lineal.

El color que domina en todas las concentraciones y motivos aislados es el rojo, excepto en la C8 donde se registran tres positivos de mano en blanco. Esta concentración es la única que presenta un caso de superposición de una mano en blanco sobre un cruciforme en rojo, por lo cual los distintos colores parecen responder a una diferencia temporal en la ejecución de las pinturas. Por otro lado, también encontramos diferencias diacrónicas si consideramos los tonos de la pintura roja. Mientras el $71 \%$ de las representaciones rojas $(n=47)$ en el Potrero de las pinturas son en tono desvaído, el $24 \%$ restante de los motivos en rojo $(n=16)$ son en tono oscuro, habiendo tres casos de motivos en rojo desvaído repintados en rojo oscuro. Todos los casos de rojo oscuro, excepto uno, y todos los casos de repintado ocurren en la C2. Esto sugiere una recurrencia en el uso de la cueva.

A lo largo del paredón, las representaciones rupestres se encuentran plasmadas entre $78 \mathrm{y}$ $239,75 \mathrm{~cm}$ de altura del suelo actual, con una media de $175 \mathrm{~cm}$ y una mediana de $190 \mathrm{~cm}$, lo cual indica que la mitad de los motivos se ubican por debajo y por encima de este valor. En la tabla 3 se presentan los valores medios, mínimo y máximo de localización de las pinturas por concentración y motivo aislado. Las concentraciones que presentan la altura media y mínima más elevada son las $\mathrm{C} 2$, C5 y $\mathrm{C} 6$, que se localizan respectivamente en la pared lateral, sobre la boca de entrada y en el vestíbulo de la cueva. Si consideramos el caso particular de la $\mathrm{C} 2$, que es la que contiene el mayor número de motivos a alturas más elevadas, y que presenta además los casos de repintado en rojo oscuro de tres motivos en rojo desvaído, se puede
Tabla 3. Altura (en cm) media, mínima y máxima de localización de las pinturas rupestres por concentración y motivo aislado en Potrero de las pinturas

\begin{tabular}{|l|c|c|c|}
\hline $\begin{array}{l}\text { Potrero de } \\
\text { las pinturas }\end{array}$ & $\begin{array}{c}\text { Media } \\
\text { aritmética }\end{array}$ & Minimo & Máximo \\
\hline C1 & 92 & 78 & 110 \\
\hline C2 & 205.51 & 180 & 212.8 \\
\hline C3 & 160 & 158 & 162 \\
\hline C4 & 181 & 160 & 205 \\
\hline C5 & 200 & 200 & 200 \\
\hline C6 & 210 & 210 & 210 \\
\hline C7 & 100.25 & 97 & 110 \\
\hline C8 & 189.43 & 144 & 203 \\
\hline MA1 & 107 & - & - \\
\hline MA2 & 215 & - & - \\
\hline
\end{tabular}

entender la localización de las pinturas en este panel en particular como un recurso de señalización del espacio.

Si evaluamos la distribución del tamaño (largo en $\mathrm{cm}$ ) de los motivos a lo largo del paredón (tabla 4), el tamaño máximo se registra en la C2 y corresponde a una serie de trazos paralelos de $41 \mathrm{~cm}$ de largo, compuesta por 13 trazos en rojo oscuro. La media de tamaño de los motivos en el Potrero de las pinturas es de $13,33 \mathrm{~cm}$, presentando el $50 \%$ de los motivos tamaños inferiores a 11,85 cm y el otro $50 \%$ tamaños más grandes.

\section{Sitios 1 y 2 en margen norte de meseta volcánica}

En el sitio 1 (S1, $70^{\circ} 25^{\circ} 13.13^{\prime \prime} \mathrm{O} 51^{\circ} 56^{\circ}$ 5.64" S), constituido por un pequeño alero en el flanco norte de la meseta, se relevaron 23 motivos distribuidos en tres concentraciones que ocupan

Tabla 4. Estadística descriptiva del tamaño (largo máximo en $\mathrm{cm}$ ) de los motivos en Potrero de las pinturas

\begin{tabular}{|l|c|}
\hline $\mathrm{N}$ & $67^{*}$ \\
\hline Mínimo & 2 \\
\hline Máximo & 41 \\
\hline Media aritmétrica & 13,33 \\
\hline Desvío estándar & 7,32 \\
\hline Mediana & 11,85 \\
\hline $1^{\circ}$ percentil $(25 \%)$ & 8,5 \\
\hline $3^{\circ}$ percentil $(75 \%)$ & 16 \\
\hline
\end{tabular}

*no se incluyen los motivos indiferenciados 
paneles con distinta orientación.

La C1 $\left(55^{\circ}\right)$ es la que presenta la mayor abundancia de motivos ( $\mathrm{n}=17)$, de los cuales un $65 \%$ $(n=15)$ son simples (tabla 1). Sólo se registran dos motivos compuestos, siendo los únicos presentes en el sitio. Se trata de un semicírculo compuesto por cuatro cruces de diferente tamaño y de un conjunto de trazos y cruces de más de 20 elementos. Los motivos abstractos son los que predominan $(n=14)$, habiendo solamente tres motivos figurativos (dos positivos de manos y un tridígito). Todos los motivos de esta concentración son en rojo desvaído. En cuanto a los aspectos técnicos, como en el caso anterior el tratamiento más frecuente es el lineal rectilíneo. Los dos casos de estampado corresponden a las manos.

La C2 $\left(340^{\circ}\right)$ solo agrupa cuatro motivos abstractos simples, tratándose de tres manchas de pintura por estarcidoy un círculo radiado de tratamiento combinado que aprovecha una oquedad del soporte para representar un círculo relleno con trazos lineales divergentes (también denominado "sol"). Todos los motivos son también en rojo desvaído.

La C3 $\left(320^{\circ}\right)$ son dos motivos simples figurativos de manos por estampado, una en rojo desvaído y la otra en rojo oscuro, lo cual sugiere diferencias temporales en la ejecución de las pinturas. $\mathrm{Al}$ igual que en la C2 del Potrero de las pinturas, el motivo en rojo oscuro se encuentra en el sector que recibe mayor insolación y presenta partes de la representación exfoliadas.

En este sitio las representaciones se ubican entre los 60 y $181 \mathrm{~m}$ de altura, con una media de 113,6 m, estando el 50\% de los motivos localizados por debajo y por arriba de los $118 \mathrm{~m}$ (mediana).

El sitio $2\left(70^{\circ} 25^{-} 6.51^{\prime \prime} \mathrm{O} \quad 51^{\circ} 56^{-} 9.28^{\prime \prime} \mathrm{S}\right)$ solo presenta tres series de trazos paralelos en rojo oscuro, dos de ellas en proximidad espacial (C1) y la tercera como un motivo aislado cortado en su base por una fisura. Las tres representaciones se ubican entre los 112 y 133 m, siendo la media de 122,5 m.

\section{DISCUSIÓN:}

\section{TENDENCIAS LOCALES Y REGIONALES}

En el Potrero de las pinturas, el sitio que concentra la mayor cantidad de motivos en la localidad, las series de trazos paralelos y las manos son los motivos predominantes. Si comparamos la distribución y abundancia de estos tipos de motivos en los sitios del flanco norte, se observa que las series de trazos paralelos están ausentes en el S1, donde se destacan las manos $(n=4)$, mientras que éstas están ausentes en el S2, donde la totalidad de los motivos son series de trazos paralelos. Los dos motivos compuestos de trazos paralelos presentes en el S2.C1 presentan trazos más largos que los del Potrero de las pinturas, con una media de 6,7 cm y valores mínimo y máximo de 5,84 y $7,27 \mathrm{~cm}$ respectivamente. En los sitios con representaciones rupestres localizados en cercanías de la laguna, no se registran manos ni series de trazos paralelos en ninguno de ellos. Sólo hay dos casos de trazos aislados y otros dos de solo dos trazos paralelos en el reparo (Gómez Otero 1983-85), es decir, en ninguna instancia se registran las largas series aquí descriptas.

En el S1 en particular, la riqueza (cantidad de clases) de motivos es alta (especialmente entre los abstractos) en relación con el tamaño de la muestra $(14 / 23)$ si lo comparamos con el Potrero de las pinturas, donde se registra prácticamente el mismo número de tipos de motivos pero en una muestra mucho más grande (15/674). Si bien este patrón, a escala local, es contrario a lo sostenido por Manzi \& Carballo Marina (2012:296), quienes señalan que en la cuenca del río Gallegos se registra una correlación positiva entre la diversidad de motivos y el tamaño de la muestra, si realizamos la prueba de Spearman considerando los datos publicados por las autoras mencionadas (Manzi \& Carballo Marina 2012:tabla 4), incluyendo los sitios de Potrok Aike, confirmamos que la tendencia regional es la por ellas señalada ( $r s=0,86453 p=0,00522$ ).

Mientras en el Potrero de las pinturas dos tipos de motivos son los que dominan los conjuntos, como lo sugiere el índice de Dominancia (tabla 5) más elevado que en el S1, en éste la abundancia por tipo de motivo es baja y se encuentra repartida de manera más homogénea (índice de Simpson de 0.904), estando la mayoría de los motivos representados por un solo ejemplar (para una explicación de los índices consultar Hammer 1999-2013 o Leonard \& Jones 1989, entre otros). La comparación estadística de los índices de Dominancia y Homogeneidad (Evenness o indice de Simpson,) entre ambos sitios ${ }^{5}$ muestra

4 No se incluyen los dos motivos indiferenciados de la C6 
Tabla 5. Comparación de distintos aspectos de la diversidad de motivos entre el Potrero de las pinturas y el sitio 1 del flanco norte

\begin{tabular}{|l|c|c|c|c|}
\cline { 2 - 5 } \multicolumn{1}{c|}{} & PP & S1 & Remuestreo $(\mathrm{p})$ & Permutación $(\mathrm{p})$ \\
\hline Riqueza & 15 & 14 & 0.998 & 0.998 \\
\hline Individuos & 67 & 23 & 0 & 0 \\
\hline Dominancia & 0.298 & 0.096 & 0.017 & 0.015 \\
\hline Índice de Simpson (Homogeneidad) & 0.702 & 0.904 & 0.017 & 0.015 \\
\hline
\end{tabular}

diferencias significativas (tabla 5). Ambos conjuntos solo comparten cinco tipos de motivos en común (a pesar que la cantidad de tipos de motivos por sitio sea similar), a saber: trazos aislados, cruciformes, circunferencias, positivos de manos y tridígitos. Un caso excepcional lo constituye la serie de trazos paralelos que no se encuentra presente en el S1 (aunque sí en el S2, excluido de la comparación, ver nota 5) y es un tipo de motivo usual a escala regional, a pesar de no ser un motivo común entre los sitios en esta comparación.

Estos aspectos apuntan una vez más hacia la diversidad que caracteriza al arte rupestre del sur de Patagonia y hacia la baja estandarización que presentan la mayoría de los motivos, especialmente los abstractos (ver también Manzi \& Carballo Marina 2012). De esta forma se registra una baja redundancia y repetición de motivos entre sitios a nivel local. Sin embargo, si se amplía la escala de comparación, las tendencias representativas son más claras, tanto en relación a la frecuencia de tipos de motivos, como así también en los procedimientos técnicos, siendo posible discriminar sectores del espacio donde los conjuntos de representaciones mantienen cierta homogeneidad interna y se diferencian de sus vecinos (Charlin \& Borrero 2012).

En el caso particular de Potrok Aike cabe señalar la ausencia de bicromías, las cuales son más frecuentes en Magallanes (Chile), combinando rojo y negro y rojo y blanco (Bate 1970, 1971). El rojo es el color dominante, tanto en la escala de la localidad como del CVPA, siendo el blanco poco frecuente. En los sitios reportados por Gómez Otero también se registra una serie tonal en negro.

Considerando las superposiciones y casos de repintado, en la escala de la localidad es posible diferenciar, de forma relativa, al menos tres momentos: el más antiguo, caracterizado por la serie tonal rojo desvaído (rojo claro en términos

5 En esta comparación se deja de lado el S2 por el pequeño de Gómez Otero), en el cual se han ejecutado la mayor cantidad de pinturas (72\% en PP incluyendo los tres casos de repintado, $96 \%$ en $\mathrm{S} 1,50 \%$ en la cueva 1 y $100 \%$ en el reparo) y dos momentos posteriores -los cuales con los datos disponibles a la fecha no es posible calibrar de manera claraindicados por un lado, por los casos de repintado en rojo oscuro de las series de trazos paralelos en rojo desvaído de la C2 de PP y por la superposición de "rojo intenso" sobre "rojo claro" en la cueva 1 (Gómez Otero 1983-85) y por el otro, por la superposición de blanco sobre rojo en la C8 de PP. Finalmente, quizás sea posible reconocer un momento aún más tardío en la serie tonal negra registrada en la cueva 1 y que se encuentra asociada con el "rojo subido", ambos colores disociados del rojo claro, registrándose asimismo un caso de superposición de "rojo subido" sobre rojo claro (Gómez Otero 1983-85). Si bien por el momento esto es especulativo, una evidencia a favor la constituye la superposición de tres trazos en negro sobre un tridígito blanco en el alero Romario Barría (Campan et al. 2007). Sin embargo, por otro lado Molina (1972:176) señala la superposición de rayas verticales rojas sobre pintura en negro en el abrigo pintado de Markatch-Aike, por lo cual la posición relativa de la serie tonal negra y su posible asociación con otros colores debe aún ser evaluada en mayor detalle.

Casos de superposición de rojo intenso sobre rojo claro también se registran en los paneles 2 y 3 de la cueva Don Ariel y de blanco sobre rojo en la cueva 5 de la "Muralla China" en la Ea. Markatch Aike (Hernández Llosas et al. 1999).

Las evidencias indirectas que han permitido asignarle una edad al "estilo Río Chico" lo asignan al Holoceno tardío. La edad más temprana registrada corresponde a Cerro Benitez 2 (Ultima Esperanza), donde se obtuvo una fecha de 2.870 265 AP (Massone 1982) en una capa que

tamaño de la muestra 
contenía lascas y rodados cubiertos de pigmento rojo. Fragmentos de ocre rojo asociados con un fogón, artefactos líticos y material faunístico fueron datados en $2.080 \pm 80 \mathrm{AP}$ en un sondeo realizado en Río Chico 1 (Bate 1970). Una fecha más tardía, de 850ะ40 AP se obtuvo en Juni Aike 1 (Gómez Otero 1989-90), donde se recuperaron granos de ocre rojo oscuro, muy similar al color de las pinturas registradas en el sitio cercano de Juni Aike 4. Estos restos fueron determinados por difracción de rayos $\mathrm{x}$ como hematina y magnetita (Gómez Otero 1989-90). Por consiguiente, los diferentes momentos de ejecución señalados en Potrok Aike deben entenderse dentro de este rango temporal, a pesar de algunos postulados previos en los que se le asigna una edad más temprana al estilo Río Chico (Bate 1970, ver discusión en Gallardo 2009).

\section{SUGERENCIAS FINALES}

El estudio sistemático del arte rupestre de Patagonia meridional aún es una tarea pendiente. Para ello se requiere del uso de un sistema clasificatorio estandarizado a la hora de hacer los relevamientos de campo y la posterior cuantificación de los motivos en laboratorio.

La necesidad de un vocabulario común en relación con la denominación de los motivos, como así también con respecto a las categorías analíticas utilizadas es una condición básica.

El estudio de la variabilidad se ve fuertemente sesgado cuando debemos agrupar diferentes tipos de motivos en clases más generales para poder realizar alguna comparación regional.

La necesidad de trabajar con datos cuantificables, ya sean frecuencias o medidas de tamaño de los motivos, resulta fundamental para establecer tendencias en una escala amplia (Tratebas 2012). En consecuencia, la unificación de los aspectos metodológicos y analíticos es el primer paso necesario antes de abordar otros problemas como la escasez de fechados y la integración de la información aportada por las representaciones rupestres con otras líneas de evidencia. Los desafíos quedan planteados y algunos intentos ya han comenzado a realizarse (Borrazzo \& Charlin 2012; Charlin \& Borrero 2012; Fiore 2006; Manzi \& Carballo Marina 2012).

\section{AGRADECIMIENTOS}

Mis agradecimientos a Luis Borrero por su lectura del manuscrito, a María Isabel HernándezLlosas por sus rápidas respuestas a mis consultas técnicas, a Julieta Gómez Otero quién también respondió numerosas preguntas sobre los antecedentes, a Mario Nuñez y personal del INTA por colaborar con nuestros trabajos en Potrok Aike y hacer grata nuestra estadía, a Thorsten Haberzettl por sus indicaciones sobre otros lugares con pinturas rupestres en Potrok Aike que motivó nuestras prospecciones y relevamientos. Estos estudios fueron financiados por el Proyecto Interdisciplinario Patagonia Austral (PIPA), PICT/ REDES $2006 \mathrm{~N}^{\circ} 02338$.

\section{BIBLIOGRAFÍA}

Aschero, C. (1979). Aportes al estudio del arte rupestre de Inca cueva-1 (Departamento de Humahuaca, Jujuy). Antiquitas, 2, 419-459.

Aschero, C., Martel, A., \& López Campeny, S. (2006). Tramas en la piedra: rectángulos con diseños geométricos en Antofagasta de la Sierra (Puna meridional, Argentina). En D. Fiore, \& M. M. Podestá (Eds.), Tramas en la piedra. Producción y usos del arte rupestre (pp. 141-156). Buenos Aires: Sociedad Argentina de Antropología, Asociación Amigos del INA y World Archaeological Congreso.

Bate, L. (1970). Primeras investigaciones sobre el arte rupestre de la Patagonia chilena. Anales del Instituto de la Patagonia, 1(1), 15-26.

Bate, L. (1971). Primeras investigaciones sobre el arte rupestre de la Patagonia chilena (segundo informe). Anales del Instituto de la Patagonia, 2, 33-41.

Belardi, J. (2004). Más vueltas que una greca. En M. T. Civalero, P. Fernández, \& A. G. Guráieb (Eds.), Contra viento y marea. Arqueología de Patagonia (pp.591-604). Buenos Aires: Instituto Nacional de Antropología y Pensamiento Latinoamericano y Sociedad Argentina de Antropología.

Borrazzo, K., \& Charlin, J. (2012). Lithic Technology, Raw Material Availability and Hunter-Gatherers Mobility: A Case Study From Southern Patagonia. Trabajo presentado en el $54^{\circ}$ Congreso Internacional de Americanistas, Universidad de Viena, Viena. MS.

Borrero, L. A., Barberena, R., Charlin, J., \& Campan, P. (2013). Geoarqueología y tafonomía en la cuenca de Potrok Aike. En J. C. Rubin de Rubin, \& R. T. 
da Silva (Eds.), Geoarqueologia (pp. 9-24). Goiás: Pontificia Universidade Católica.

Campan, P., Carballo Marina, F., \& Manzi, L. (2007). Arqueología de Estancia La Carlota (Campo Volcánico Pali Aike, Argentina). En F. Morello, M. Martinic, A. Prieto, \& G. Bahamonde (Eds.), Arqueología de FuegoPatagonia. Levantando piedras, desenterrando huesos... y develando arcanos (pp. 687-700). Punta Arenas: CEQUA.

Charlin, J. (2009). Estrategias de aprovisionamiento y utilización de las materias primas líticas en el campo volcánico Pali Aike (Prov. Santa Cruz, Argentina). Oxford: Archaeopress.

Charlin, J., \& Borrero, L. (2012). Rock Art, Inherited Landscapes And Human Populations In Southern Patagonia. En J. McDonald, \& P. Veth (Eds.), A Companion to Rock Art (pp. 381-398). USA-UK: Wiley-Blackwell.

Charlin, J., \& Pallo, M. C. (2013). Disponibilidad de materias primas líticas y uso del espacio en el interfluvio Gallegos-Chico (Pali Aike, Santa Cruz, Argentina). En F. Zangrando, R. Barberena, A. Gil, G. Neme, M. Giardina, L. Luna, C. Otaola, S. Paulides, L. Salgán, \& A. Tivoli (Eds.), Tendencias teórico-metodológicas y casos de estudio en la arqueología de la Patagonia (pp. 307-316). Buenos Aires: Sociedad Argentina de Antropología.

Fiore, D. (2006). Poblamiento de imágenes: arte rupestre y colonización de la Patagonia. Variabilidad y ritmos de cambio en tiempo y espacio. En D. Fiore, \& M. M. Podestá (Eds.), Tramas en la piedra. Producción y usos del arte rupestre (pp. 43-62). Buenos Aires: Sociedad Argentina de Antropología, Asociación Amigos del INA y World Archaeological Congress.

Gallardo, F. (2009). Sobre la composición y la disposición en el arte rupestre de Chile: consideraciones metodológicas e interpretativas. Magallania, 37(1), 85-98.

Gómez Otero, J. (1983-1985). Investigaciones arqueológicas en las cuencas media y superior del río Gallegos. Informe final de beca de perfeccionamiento. Buenos Aires: Conicet. MS.

Gómez Otero, J. (1986-1987). Investigaciones arqueológicas en el alero Potrok-Aike (Provincia de Santa Cruz). Relaciones, XVII(I), 173-198.

Gómez Otero, J. (1987). Posición estratigráfica particular de puntas de los períodos IV y $\mathrm{V}$ de Bird en el alero Potrok-Aike (Santa Cruz). En Primeras Jornadas de Arqueología de la Patagonia (pp. 125-130). Chubut: Dirección de Cultura de la Provincia del Chubut.

Gómez Otero, J. (1989-1990). Cazadores tardíos en la zona fronteriza del paralelo $52^{\circ}$ sur. El paraje de Juni Aike. Anales del Instituto de la Patagonia, 19, 47-71.

Gómez Otero, J. (1993). The function of small rockshelters in the Magallanes IV phase settlement system (South Patagonia). Latin American Antiquity, 4(4), 325345.

Gradín, C. (1978). Algunos aspectos del análisis de las manifestaciones rupestres. Revista del Museo Provincial, I(1), 120-133.

Hammer, Æ. (1999-2013). PAleontological STatistic. Version 3.01. Reference Manual. http://folk.uio.no/ ohammer/past/

Hernández Llosas, M. I. (1985). Diseño de sistema de documentación para representaciones rupestres. En PROINDARA: Programa de Investigación y Documentación de Arte Rupestre Argentino (pp. 9-65). Buenos Aires: Instituto de Antropología e Historia Hispanoamericanas.

Hernández Llosas, M. I., Nami, H., \& Cuadrado Woroszylo, M. (1999). Arqueología en la localidad arqueológica de Pali Aike, cuenca del río Chico. II. Resultados preliminares sobre las representaciones rupestres. Praehistoria, 3, 202-217.

Leonard, R., \& Jones, G. (Eds.). 1989. Quantifying Diversity in Archaeology. Cambridge: Cambridge University Press.

Manzi, L., \& Carballo Marina, F. (2012). Manifestaciones rupestres en el campo volcánico Pali Aike (cuenca del río Gallegos, Santa Cruz, Argentina). Magallania, 40(1), 283-302.

Massone, M. (1982). Nuevas investigaciones sobre arte rupestre de Patagonia meridional chilena. Anales del Instituto de la Patagonia, 13, 73-94.

Menghin, O. (1952). Las pinturas rupestres de la Patagonia. Runa, V(1-2), 5-22.

Menghin, O. (1957). Los estilos de arte rupestre de Patagonia. Acta Praehistorica, 1, 57-87.

Molina, M. (1969). Arqueología patagónica.- Arte rupestre austral. Antiquitas, IX, 24-30.

Molina, M. (1969-1970). El abrigo de los Pescadores. Anales de Arqueología y Etnología, 24/25, 239-250.

Molina, M. (1972). Nuevos aportes para el estudio del arte rupestre patagónico. Anales de la Universidad de la Patagonia "San Juan Bosco", 4, 64-182.

Molina, M. (1976). Patagónica. Prehistoria, Tradiciones y Mitología. Comodoro Rivadavia: Librería Ateneo Saleciano, Universidad de la Patagonia "San Juan Bosco". 
Prieto, A. (1989-1990). Cazadores tardíos en la zona fronteriza del paralelo $52^{\circ}$ sur. El alero Peggy Bird. Anales del Instituto de la Patagonia, 19, 73-85.

Prieto, A. (1997). Algunos resultados de los trabajos arqueológicos en Juni Aike 2. Anales del Instituto de la Patagonia, 25,137-146.

Prieto, A., Morello, F., Cárdenas, P., \& Christensen, M. (1998). Cañadón Leona: A sesenta años de su descubrimiento. Anales del Instituto de la Patagonia, 26, 83-105.

Rohlf, F. J. (2010). tpsDIG2. version 2.16. Department of Ecology and Evolution, State University. Stony Brook, New York. http://life.bio.sunysb.edu/morph/

Sanguinetti de Bórmida, A. (1976). Excavaciones prehistóricas en la cueva de Las Buitreras, Santa Cruz, Argentina. Relaciones, X, 271-292.

Sanguinetti de Bórmida, A. (1982). Introducción a la prehistoria de la Patagonia, Argentina. Tesis doctoral. Facultad de Filosofía y Letras, Universidad de Buenos Aires. Buenos Aires. MS

Selpúlveda, M. (2011). Pinturas rupestres y tecnología del color en el extremo sur de Chile. Magallania, 39(1), 193210

Tratebas, A. (2012). North American-Siberian Connections: Regional rock art patterning using multivariate statistics. En J. McDonald, \& P. Veth (Eds.), A Companion to Rock Art (pp. 143-159). USA-UK: Wiley-Blackwell. 